\title{
Role of birth weight and predisposition for adverse body composition in early childhood: Findings from the ROLO study
}

\author{
Aisling A. Geraghty ${ }^{1}$, Eileen C. O’Brien ${ }^{1}$, Mary Horan ${ }^{1}$, Jean Donnelly ${ }^{1}$, Eleanor Molloy ${ }^{2}$ and \\ Fionnuala M. McAuliffe \\ ${ }^{1}$ UCD Perinatal Research Centre, School of Medicine, National Maternity Hospital, University College Dublin, \\ Dublin, Ireland and \\ ${ }^{2}$ Discipline of Paediatrics, Trinity College Dublin, Dublin, Ireland
}

\begin{abstract}
Introduction: The early fetal environment during pregnancy is extremely important and research indicates that weight at birth can have crucial impacts for the individual's health later in life. With rates of childhood obesity estimated to be as high as $21 \%$ in some European countries, it is vital that early risk factors are identified so that interventions can be developed. We aimed to investigate if children born macrosomic (birth weight $>4 \mathrm{~kg}$ ) remained larger than normal birth weight babies up to 5 years of age.

Materials and Methods: This is a longitudinal follow-up of 387 five-year-old children (53\% born with macrosomia, 47\% normal birth weight) born into the ROLO randomised control trial in the National Maternity Hospital, Dublin (ISRCTN54392969). Birth weight was previously recorded then at 6 months, 2 years, and 5 years of age child height, weight, anthropometric and skinfold measurements were collected. Body Mass Index $\left(\mathrm{kg} / \mathrm{m}^{2}\right)$ and centiles were calculated. Student t-tests and Mann-Whitney U tests were used to compare the two groups with multiple linear regression modelling to control for confounders.
\end{abstract}

Results: Children with a birth weight $>4 \mathrm{~kg}$ had consistently higher weights, lengths, and BMI centiles, along with increased head and chest circumferences, compared to normal birth weight children from 6 months up to 5 years of age ( $p<0.05)$. After controlling for child sex, intervention group, smoking during pregnancy, maternal education status, and maternal BMI, children with macrosomia were $0.61 \mathrm{~kg}$ heavier than non-macrosomic infants at 5 years of age $(95 \% \mathrm{CI}: 0.04-1.18, \mathrm{p}<0.05)$.

Discussion: Children born with a high birth weight remain heavier and larger into childhood. These individuals are at a higher risk of obesity which highlights the need for monitoring and potential interventions, both during pregnancy and in infancy, to curb the current childhood obesity crisis.

\section{Conflict of Interest}

There is no conflict of interest 